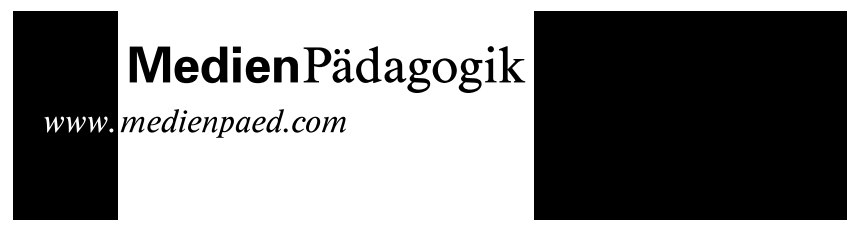

Jürgen Oelkers

22.5.2000

\section{Schule und Bildung im Prozess der Globalisierung}

Seit ihren Anfängen waren Schulen immer lokal, also ortsgebunden und in diesem Sinne geschichtlich. Die Konzepte dagegen, die Theorie der Schule oder die Flügel der Gedanken, waren spätestens seit Comenius immer global. Daraus entsteht die schöne Paradoxie, dass global - überall auf der Welt - verwirklicht werden soll, was nur seinen bestimmten historischen Platz haben kann, mithin überall verschieden sein muss. «Global» ist damit zugleich eines und verschiedenes, es ist an einem Ort und an allen. Comenius spricht in der Pampaedia, also dem Zentrum seiner Bildungstheorie, von «omnes - omnia - omnino», alle Menschen werden durch das Ganze und so durch Alles von Grund aus zur Vollkommenheit geführt (Komensky 1970, S. 15), was nur denkbar ist, wenn Bildung als globale Kraft angesehen wird, die an jedem Ort alle erreicht, und dies auf gleiche Weise. Aber jeder Ort hat andere Schulen, es gibt kein pädagogisches «überall gleich», die Geschichten sind ebenso verschieden wie die künftigen Entwicklungen. «Globalisiert» sind nur die Ideen der perfekten Bildung, «Vollkommenheit» für alle und in allem ist daher nicht Ziel, sondern nur Wunsch, der Gleichheit im letzthin Möglichen sucht und dabei die Realitäten überspringen kann.

Heute wird «Globalisierung» als Realität verstanden, auf die Erziehung und Bildung reagieren sollen und müssen. Was als Realität angenommen wird, ist zumeist diffus bezeichnet, irgendwie wird vom Internet auf die Welt geschlossen oder von Aktienkursen auf Bildung, die gelegentlich so dargestellt wird, dass sie eigentlich nur noch auf eine feindliche Übernahme warten kann. Schlagworte kursieren besonders dann erfolgreich, wenn die Realität weitgehend Behauptung ist und noch nicht oder nicht mehr Erfahrung. Die Paradoxie bleibt bestehen: Bildung, mindestens Schulbildung, ist eine ortsgebundene Erfahrung, solange die Schulen sich nicht ins Internet aufgelöst haben, müssen Kinder jeden Morgen während der staatlich fixierten Schulzeit an einem bestimmten Ort zur Schule gehen, die sie nicht ohne Hintersinn für Thema als ihre Schule bezeichnen. Es ist eine Örtlichkeit, nicht viele, es sind bestimmte Personen, nicht alle, es sind unwiederholbare Erfahrungen, nicht beliebige. In diesem Sinne ist Schule mit einer lokalen Identifizierung verbunden, die sich nicht global ausweiten lässt, während die Globalisierung nach einer ihr angemessenen Bildung verlangt, ganz so könnte Comenius' Phantasie der Allheit doch noch verwirklicht werden.

Die internationale Bildungspolitik ist in dieser Hinsicht unzweideutig: Es gibt nicht ein nationales Bildungsssystem, das nicht unter politischem Druck steht, auf «Globalisierung» reagieren zu müssen. Meist soll möglichst schnell möglichst viel geschehen, kommuniziert mit eindrucksvollen Formeln, die zumeist nicht sagen, wie sich das Leitthema «Globalisierung» in ein Feld übersetzen lässt, das nicht einfach auf «die Welt» - und sei sie noch so verändert - reagieren kann. Die Formeln sind solche der Expertenkommunikation, die sich auf fast ironische Weise global verbreiten kann, nämlich zwischen Washington, Tokio, London und der Schulaufsicht in Oberbayern nahezu zeitgleich entsteht, nur schwach übersetzt zu werden braucht und doch einen Gegenstand gar nicht hat. Selbst wenn Experten wüssten, was globalisierte Bildung oder Globalisierung der Bildung bedeutet, wären immer noch die Voraussetzung einer, wie es neudeutsch heisst, «Implementation» der Konzepte hochgradig differente nationale Systeme und letztlich Varianz bis hinab zur Einzelschule, die ja nicht ohne Grund zum eigentlichen Adressaten der Bildungsreform geworden ist. Dann aber ist es mehr als ironisch, von den Volksschulen einen Beitrag zur Globalisierung der Bildung zu erwarten, die zunächst nur als Formel erscheint.

Dennoch stellt sich das Thema, unabhängig von der Frage, wie ironisch ich als Experte nun selber eigentlich werden müsste. Aber es stellen sich mindestens drei ernsthafte Probleme, die ich im Folgenden bearbeiten werde: Zunächst sind Globalisierungsfolgen im Alltagsleben sichtbar und so ein Faktor der Erziehung (1). Zweitens sind die Bildungssysteme zum ersten Mal seit Beginn des 19. Jahrhundert einem technischen Innovationsschub ausgesetzt, der sie nachhaltig verändern wird (2). Und drittens stellen sich neue Fragen der Legitimation, und zwar weil oder soweit die neuen technischen Möglichkeiten, einhergehend mit sozialen und kulturellen Umbrüchen, den bisher geltenden Generationenvertrag «Bildung» in Frage 
stellen (3). Was also diskutiert werden muss, ist nichts weniger als die Frage, ob Volksschule und öffentliche Bildung in einer medial radikal gewandelten und sozial grundlegend sich ändernden Umwelt noch tragfähige Konzepte sind.

\section{Globalisierungsfolgen}

Was heute unter dem Stichwort «Globalisierung» diskutiert und erfahren wird, hat mindestens die folgenden Komponenten,

- die Auflösung nationaler und so geschützter Arbeitsmärkte,

- die Digitalisierung der Produktion,

- die Standortunabhängigkeit von Betrieben und Unternehmen,

- die Verdichtung weltweiter angelsächsischer Kommunikation,

- die Erweiterung und Vernetzung aller Abhängigkeiten und Risiken, der Rückgang oder die Verlagerung des Sozialen und

- die Standardisierung des Ästhetischen.

Paradoxerweise wird dadurch auf historisch völlig neue Weise Individualisierung befördert, versteht man darunter Selbstinszenierung und Selbstverantwortung. Nicht der Betrieb sorgt mehr für seine Mitarbeiter, diese sorgen für sich, möglichst so, dass sie selber Unternehmer werden. Das ist möglich, weil die Risiken neu verteilt werden. Die kollektiven Vorsorgen des 19. Jahrhunderts werden individualisiert, ohne umgekehrt Individualisierungen, zum Beispiel solche, die auf zunehmende Lebensdauer reagieren, neu mit kollektiven Massnahmen bearbeiten zu können. Die Altersvorsorge muss unabhängig von familialen Netzen organisiert werden, je nach individuellen Möglichkeiten und in der Hoffnung, möglichst lange für sich unabhängig leben zu können. Echte Pflegeplätze sind unbezahlbar, wobei die Angehörigen Vorsorge für den Fall treffen müssen, wenn das Gesetz sie in die Verantwortung nimmt.

Eine zumeist nicht beachtete Globalisierungsfolge ist der Zwang zur Jugendlichkeit, der die gesamte Imagesteuerung des Alltagslebens beherrscht. Die Ästhetik des Jungen prägt paradoxerweise vor allem Alte, die optisch nachweisen müssen, dass sie jung genug aussehen und sich diesem Aussehen entsprechend verhalten, um ästhetische Akzeptanz erwarten zu können. Das geschieht weltweit auf allen Kommuniationsmärkten und lässt nur solche Varianz zu, die das Grundimage bestärkt. Talkshows für Alte also Shows in den ungünstigsten Sendezeiten - müssen jung aussehen. Wie stark dieser Zwang ist, lässt sich auch daran ablesen, dass Fünfund- vierzigjährige als «ältere Arbeitnehmer» bezeichnet werden können, so wie eine Ehe eine «Altehe» ist, wenn sie nach fünfzehn Jahren immer noch besteht. Schliesslich gilt «Kreativität» als jugendlich, wer «produktiv» sein will, muss zugleich kreativ und jung sein, das Image erfolgreicher Computerfirmen ist entsprechend immer schnell, dynamisch und jung. Aussehen und auch Verhalten verbrauchen sich auf Erneuerung hin. Die Lebenszeit wird auf Abschnitte hin berechnet, grosse Kontinuitäten sind zunehmend die Ausnahme, in diesem Sinne heisst Globalisierung auch im Alltagsleben Wechsel, Flexibilisierung, Stress und Suche nach Entlastung. Die festen Orte verschwinden, wenigstens solche, die auf eine ganze Lebensspanne hin berechnet sind. Das gilt nicht nur für die Börsenkurse, die gesamte Produktion wird zunehmend unabhängig vom Ort der Fabrik, und dies nicht nur im Sinne einer just-in-time-Produktion, bei der Zulieferer dafür sorgen müssen, dass das Material den Ort der Herstellung zum genau bestimmten Zeitpunkt erreicht. Die Herstellungsorte selber werden flexibel, was nicht in Hamburg gebaut werden kann, wird nach Hongkong verlagert, mit der Pointe, dass zunehmend weniger wirklich gebaut werden muss. Der Arbeitsmarkt reagiert also immer weniger auf Produktion, sondern auf Kommunikation, die weltweit geführt werden muss. Nicht zufällig sind Internet-Foren für die Jobsuche heute vielversprechender als Arbeitsämter, was nochmals zeigt, wie ganze alte Ordnungen innerhalb kürzester Zeit wegbrechen können. Das gilt mit Nachdruck, wenn ich das so sagen darf, auch für die sozialen Ordnungen, und zwar unabhängig davon, ob sie öffentlich oder privat verfasst sind. Dieser Gegensatz des 18. Jahrhunderts verliert offenbar an Bedeutung, wenn Intimes Showthema werden kann oder persönliches Leid zur Inszenierung öffentlicher Anteilnahme verwendet wird. Was auf diese Weise entsteht, öffentliche Geschwätzigkeit, ist unausweichlich, und darum mit der Dauer immer weniger abstossend. Widerstand wird durch Gewöhnung ersetzt, auch weil sich die Konsummedien dem Protest entziehen. Ihr Massstab ist nicht Geschmack, sondern Quote, und dies weltweit.

Ein Basisvorgang ist die Flexibilisierung von Zeit. Arbeit wie Produktion lassen sich nicht mehr an Stundenbudgets fesseln, die Korrelation von Arbeitszeit, berechnet in Stunden, und Produktivität bricht auseinander, weil die Arbeitsvorgänge oder Tätigkeiten immer mehr an festen Standards verlieren. Mit der Fabrik verschwindet zugleich das Fliessband und so der Arbeitstakt. Das Problem ist nicht mehr, den Arbeitstakt zu steigern oder abzusenken, sondern ohne Takt arbeiten zu müssen, mit einer individuell 
berechneten Zeit, die vom Ergebnis her kalkuliert wird. Wie viel Zeit für das einzelne Produkt angenommen werden muss, ist nicht in einem allgemeinen Sinne klar, sondern wird individualisiert, je nach der Leistungsbereitschaft oder -fähigkeit einzelner Mitarbeiter. Der Vergleichsstandard ist nicht mehr die Wochenarbeitszeit, sondern das Produkt, entsprechend individualisiert wird der Wettbewerb, mit allen Folgen einer instabilen Belohnung, die sich vom persönlichen Erfolg oder Misserfolg her berechnet, nicht nach Tarifverträgen, die unabhängig von persönlichen Leistungsunterschieden gelten und für alle Arbeitnehmer einer Branche festgelegt sind. Derartige Zeitmargen verschwinden mit der Individualisierung der Arbeit, die die Fesseln der Industrialität überwinden wird oder längst überwunden hat.

Damit sind soziale Güter ersten Ranges bedroht, nämlich arbeitsunabhängige Zeiten, die ihren Reservatcharakter verlieren. Geschäftliche EMails kann man um 3.00 Uhr nachts genauso senden oder empfangen wie am Samstag Mittag, ähnlich sind Websites an keine Öffnungszeiten gebunden und kann ein Internetservice in einem buchstäblichen Sinne rund um die Uhr abgerufen werden. Dabei werden schnelle Antworten auf eilige Anfragen erwartet, die auf Arbeitszeiten keine Rücksicht nehmen können oder müssen. Man reagiert gereizt auf jede Störung des Computers, der als verstörendes Schicksal erscheinen kann, weil wertvolle Zeit verloren geht, die anders eingeplant war. Eine wesentliche Errungenschaft der Gewerkschaftsbewegung im ausgehenden 19. und beginnenden 20. Jahrhundert, die strikte Trennung zwischen Arbeit und Freizeit, verliert ihren institutionellen Charakter. Ein Arbeitstag am Computer beginnt nicht um 9.00 Uhr und endet um 17.00 Uhr, er beginnt und endet überhaupt nicht, vielmehr kann immer nur eine fortlaufend bestehende Arbeitsmöglichkeit unterbrochen werden, mit individuell kalkulierten Pausen, die auf Belohnungsdruck ebenso reagieren müssen wie auf die Schnelligkeit der Konkurrenz. Das schafft zum Beispiel völlig neue Probleme, wie Eltern die Zeit der Erziehung kalkulieren wollen oder müssen, was nicht einfach dadurch möglich ist, dass Kinder möglichst schnell selbst am Computer arbeiten. Paradoxerweise gibt es mit der Auflösung der strikten Trennung zwischen Arbeit und Freizeit zunehmend weniger Möglichkeiten, der Freizeitindustrie auszuweichen, die ihrerseits rund um die Uhr anbietet und tatsächlich alle Interessen und Instinkte zu bedienen versteht. Das gelingt umso mehr, je genauer dafür Zeit eingeplant werden muss. Ein «Sonntag» verliert den Charakter stabiler Wiederholung, Rituale entlasten nicht mehr, vielmehr belasten ständig ausgetauschte Ansprüche, die wie Wunscherfüllungen erwartet werden. Individualisierte Freizeitrhythmen sind immer zugleich Erwartungen, dass noch ganz andere Wunscherfüllungen möglich sind, abhängig davon, wie erfolgreich die Erwerbszeit gestaltet wird. Das geschieht zunehmend autonom, wenngleich nicht unabhängig, weil Aufträge erfüllt werden müssen und Abgabetermine gesetzt sind. Wie bedrohlich diese neue Freiheit unterschwellig wahrgenommen wird, zeigt sich daran, dass Lottogesellschaften lebenslange Renten als Hauptgewinne anbieten, die die Glücklichen von den Beladenen unterscheiden können.

Eine Folgenabschätzung wäre auch vor Etablierung des Systems kaum möglich gewesen, mit der Etablierung aber ist sie folgenlos oder zieht sich in die Didaktik zurück. Darüber gleich mehr. An dieser Stelle muss noch auf die pädagogischen Folgen von Globalisierungsprozessen hingewiesen werden. Im öffentlichen Diskurs ist zumeist die Rede von «Scheidungskindern», «Nintendo-Kids», «Wertezerfall» oder zunehmender «Verwahrlosung» unter den Verhältnissen einer Wohlstandsgesellschaft. Aber das sind entweder keine oder schwache Kausalitäten, mit denen sich zudem der historische Wandel kaum sehr weit erfassen lässt, und sei es nur aus dem Grunde, dass derartige Klagen die Geschichte der Erziehung begleiten. Aber für den heutigen Wandel ist die Geschichte nicht der Massstab, weil historische Beispiele, für das, was geschieht, fehlen. Das lässt sich an mindestens fünf Faktoren zeigen, die das Feld der Erziehung grundlegend verändert haben und weiter ändern werden:

1. Die Entgrenzung der Erziehungszeit

2. Die Kommerzialisierung der Kindheit.

3. Die Verlagerung von Risiken auf die Kinder.

4. Die Emanzipation beider Eltern.

5. Die fortlaufende Korrektur der Lebensentwürfe.

Dass Erziehungszeit knapp ist und mit der Knappheit nach der Qualität beurteilt wird, ist eine Erfahrung, die früheren Kindergenerationen fremd war. «Knappheit» ist nicht einfach Dosierung der Zuwendung, sondern Kalkulation der Anwesenheit und mit der Anwesenheit der Aktivität. Erziehungszeit verstreicht so nicht einfach, sie muss gestaltet werden und wird an der Gestaltung gemessen, und zwar sowohl von den Kindern wie von der beobachtenden Umwelt. Die Bedingung ist, dass weit weniger Zeit zur Verfügung steht als Gestaltungsmöglichkeiten abverlangen, was sofort sichtbar wird, wenn man beobachtet, wie gehetzt Kindergeburtstage bei 
McDonald's absolviert werden, wie lang die Wege sind zwischen dem Klavier- und dem Ballettunterricht oder wie weit der Abstand zwischen der Wohnung und einem Spielplatz ist, der halbwegs akzeptabel erscheint. Kinder unterscheiden zum Beispiel Schwimmbäder danach, welchen Komfort das Wellenbad bietet, was Eltern in die Verlegenheit setzen kann, der Präferenz ihrer Kinder nur mit einem hohen Zeitaufwand erfüllen zu können, der andere Aktivitäten ausschliesst. «Qualität» bieten nur bestimmte Angebote, so dass auch Kindheit nach events organisiert wird, besonderen Ereignissen, die nicht mehr selten sein dürfen.

Kindheit ist kommerziell geworden, in einem Ausmass, das die früheren Bleyle-Kalender als naive Kindorientierung erscheinen lassen muss. Kinder sind nur Objekt, sondern zugleich Subjekt von Markenwerbung, sie entwickeln früh «Markenbewusstsein», also können zwischen Logos vergleichbarer Produkte unterschieden und eigene Präferenzen festlegen, Kinder artikulieren Konsumwünsche und erhalten sie mehr oder weniger passgenau erfüllt, sie setzen Medienzugang durch und nutzen die knappe Zeit ihrer Eltern, um ihre Unabhängigkeit zu vergrössern. Im Gegenzug werden Risiken übertragen, Kinder sind für ihre eigenen Lebensentwürfe auch dann zuständig, wenn sie keine haben, Kinder sind mit Beziehungsproblemen mehr oder weniger direkt belastet, für psychische Schäden ist niemand zuständig, irgendeine einklagbare pädagogische Verantwortung besteht im Normalfall der Erziehung nicht, was auch daran abgelesen werden kann, dass das materielle Erbe nicht mehr quasi-automatisch auf die Kinder übergeht. Das hängt mit Emanzipationsentwürfen zusammen, die nicht über das individuelle Leben hinausreichen. Selbstverwirklichung wird nicht mehr auf die Kinder projiziert, mit allen Folgen für den Status der Kinder in der Lebensplanung ihrer Eltern. Sie sparen nicht länger, und zwar weder Moral noch Geld, für die kommende Generation, die entsprechend früh als unabhängig definiert werden kann, entlassen sozusagen in eine Wirklichkeit, die von den Kindern noch weit mehr als von den Eltern die ständige Korrektur der Lebensentwürfe abverlangt.

Auf eine solche produktive und riskante Instablilität ist weder Erziehung noch Bildung eingestellt. Mindestens in ihren theoretischen Selbstbeschreibungen betonen sie die langfristige, heute sagt man nachhaltige Ausrüstung mit Moral oder Wissen, die möglichst bis zum Ende des Lebens vorhalten soll. Ich kenne keine Theorie der Erziehung, die davon ausgeht, dass Kinder in ihrem späteren Leben ihre moralische Identität mehrfach neu und anders bestimmen müssen, ohne einfach Anschlüsse suchen zu können, also mit hohen Verlustrisiken. Allein das macht die Idee der «Ausrüstung mit Sittlichkeit» fraglich und wirft neues Licht auf den Anspruch möglichst früh einsetzender Erziehung, die ja gedacht wird, als führe ein gerader Weg ins Leben als Erwachsener. Aber das Leben künftiger Generationen folgt nicht mehr einem grundlegenden Entwurf, der nicht früh genug gefasst werden kann. Dafür fehlen alle Stabilisatoren, fest umrissenene Berufe, unantastbare Beziehungen oder auch das Denken in Kindern, also mit Zukünften über das eigene Leben hinaus. Zukunft wird daher nicht nur verzeitlicht, sie wird individualisiert, also begrenzt auf das, was innerhalb des eigenen Erwartungshorizontes absehbar und erreichbar erscheint. Bildung im Sinne der Volksschule kann so nicht gedacht werden. In einem zweiten Schritt gehe ich näher auf das ein, was bestimmte Autoren «globalisierte Bildung» oder «Globalisierung der Bildung» nennen. Die Legitimation der Volksschule wird wesentlich davon abhängen, was darunter genau zu verstehen ist, und vor allem, was darunter nicht zu verstehen ist.

\section{Wandel der Bildungssysteme}

Ob es eine politische Weltdemokratie via Internet geben wird oder nicht, ist für die Frage der Bildung nicht vorrangig. Was durch die Medienrevolution herausgefordert wird, ist die nationalstaatliche Organisation und so der Schutz oder die Privilegierung von Bildung, die im 19. Jahrhundert entstanden ist und alle bisherigen Medienrevolutionen erfolgreich abwehren konnte (Cuban 1986). Aber Internet und Computer sind kein zweiter Fall «Sprachlabor» und «programmiertes Lernen», also technische Innovationen, die mit hohem Aufwand eingeführt wurden und unbeachtet bleiben konnten. Was das Internet ändert, ist mindestens dreierlei, die Zugänge zum Wissen können nicht mehr auf das Format des Schulbuchs beschränkt werden, das Wissen selbst kann beliebig angereichert werden und die Kontrolle ist nahezu unmöglich. Das gilt für alle Stufen des Systems, auch Universitätsprofessoren können die tatsächliche Herkunft einer Seminararbeit nicht prüfen, es sei denn, sie sind ständig im Netz. Studierende, im Übrigen, werden in Zukunft weltweit anrechenbare Qualifikationsleistungen einholen können, und es bleibt abzuwarten, ob dies wirklich bei den Besten jeden Faches geschieht, die schon aus Gründen der Kapazität den Zugang drastisch beschränken müssen. Man kann zwei Anfragen am Tag beantworten, aber nicht zweihundert. 
Wesentlich sind aber ganz andere Folgen, die mit dem historischen Typus Verschulung zu tun haben. Schulen sind, ich wiederhole diesen Punkt, ortsgebunden, und das hat unmittelbare Konsequenzen für die Prinzipien der Schulorganisation, die seit dem ausgehenden Mittelalter in Kraft sind und deren Geltung bislang völlig fraglos zu sein schien. Schulen sind individuell immer nur im Rahmen einer bestimmten Form, die universell ist. Das gilt für die Ästhetik des Unterrichts ebenso wie für die Verteilung der Rollen oder die Fragilität des Kollegiums und die Mentalität «Lehrersein». Interessant im Blick auf Globalisierungsfolgen sind die Selbstverständlichkeiten der Schulorganisation, also mindestens die folgenden Prinzipien:

1. Alle Schüler einer Lehr- und Lerneinheit sind während der Unterrichtszeit persönlich anwesend.

2. Lehren und Lernen ist auf anwesende Personen abgestellt, Abwesenheit ist Chancenminderung

3. Lernobjekt und Lernzeit sind pro Gruppe oder Einheit genau gleich.

4. Es gibt nur ein Zeitmass.

5. Alle Medien dienen dieser Organisation.

Globalisierung in diesem Sinne heisst nicht nur Erreichbarkeit an jedem Standort der Welt, nicht nur Abrufbarkeit jeder Information, nicht nur Kombinationsfähigkeit jedes Elements, sondern zugleich Unberechenbarkeit bei Verlust von Rhythmus und kollektivem Lebensgefühl. Das Ganze wird nicht, um einen Slogan aus den achtziger Jahren zu zitieren, unübersichtlich, auf paradoxe Weise schliesst Globalisierung jegliche Vorstellung des Ganzen überhaupt aus. «Unübersichtlichkeit» ist gegeben, wenn und soweit ein höherer Standpunkt fehlt (Oelkers 1999), aber Globalisierung bezieht sich nicht auf eine Ganzheit, die nur nicht hoch genug überschaut werden kann, vielmehr schliesst die Vernetzung aus, dass ein Ganzes erscheinen kann. Das Internet ist keine Ganzheit, sondern eine ständig sich erweiternde Vernetzung, deren Grösse sich der menschlichen Raumvorstellung entzieht. Ganzheiten müssten abgeschlossen sein, während Vernetzung heisst, dass auch die Grenzen des Ganzen verschwinden, so paradox das klingen mag. «Globalisierung», könnte ich auch sagen, überwindet den Globus, auch weil darunter keine konkreten Prozesse wie die Entdeckung Amerikas und deren Folgen mehr verbunden sind. Globalisiert werden kommunikative Netze, Datensätze, Steuerungssysteme, die nicht mehr mit dem Kontakt haben, was im Alltagsleben als «Realität» erscheint. Umso mehr ist diese Realität von den Globalisierungsfolgen bestimmt, die ja nicht qua Abstimmung abgewandt oder per politischem Entschluss befördert werden können. Man kann, was freilich auf rasante Weise immer unwahrscheinlicher wird, das Internet nicht nutzen, aber das hebt die Realität Internet nicht auf. Im Übrigen ist die Einführung nie politisch abgestimmt worden, sie hat einfach stattgefunden, unabhängig vom politischen Willen, der sich im Blick darauf gar nicht artikuliert hat und offenbar zu haben brauchte.

Die Umstellung auf computerorientiertes Lernen bricht den unbedingten Vorrang der Personen auf, Lernen kann sich auch auf Programme beziehen, für die persönliche Anwesenheit nicht mehr zwingend erforderlich ist. Zudem sind ganz andere Betreuungsverhältnisse denkbar, Internetlehrer gibt es bereits heute schon, sie verlangen nicht, dass alle ihre Schüler zur gleichen Zeit und in der gleichen Situation lernen, wie die Schulorganisation dies verbindlich vorschreibt, unabhängig davon, wie die tatsächliche Lernmotivation beschaffen ist. Wieder würde sich eine soziale Organisationsform auflösen, zugunsten einer individuellen Nutzung von Zeit und Technologie. Wesentlich dabei ist, dass Zeit flexibilisiert wird. Lernen ist nicht in starre Zeiteinheiten, Lektionen oder Schulstunden, kanalisiert, sondern kann und muss sich selbst organisieren, eine analoge und passende Flexibilisierung der Betreuung vorausgesetzt. Unterricht wäre dann nicht mehr lediglich Lehrertätigkeit, die auf Aufnahme des akut Vorgebrachten abhebt, vielmehr würden sich Aufgaben und Leistungen zeitlich trennen. Die Leistung von Schülern ist dann nicht mehr identisch mit dem, was in der einen und grundlegenden Lernsituation vorhanden ist und wahrgenommen wird, abhängig von dem, was die Lehrkraft darstellt oder abverlangt.

Abwesenheit ist nicht länger gleichbedeutend mit Chancenminderung, weil die Grundbedingung, Anwesenheit von allen, aufgebrochen ist. Aufgaben können verschieden bearbeitet werden, und die Leistungskontrolle setzt nicht mehr eine ununterbrochene Kette von Situationen voraus, die sämtlich von der einen Lehrkraft gestaltet wurden. Sie ist verantwortlich für Lernerfolg, nicht dafür, dass alle Schüler exakt das aufnehmen, was zufällig sie geboten hat. Die Schüler nutzen die medialen Zugänge zur Bearbeitung von Aufgaben, sie werden damit unabhängig von dem Wissensgefüge und dem Lehrstil eines und ihres Lehrers, der sich mit den Zugängen zu den Medien unweigerlich einer Konkurrenz ausgesetzt sieht, die früher durch das verpflichtende und vom Lehrer kontrollierte 
Schulbuch ausgeschlossen oder kurzgehalten werden konnte. Aber will oder kann man es Schülern verweigern, sich die notwendigen Informationen zur Bearbeitung ihrer Aufgaben weltweit zu beschaffen? Niemand kann kontrollieren, ob Semesterarbeiten aus dem Internet stammen oder das Produkt eigener Arbeit sind, mit der Pointe, dass ein Mix aus Internet und eigenem Kopf erwartbar ist, der vermutlich in vielen Fällen des Lernen befördern wird.

Die Medien dienen also nicht mehr der Schule, wie das Schulbuch der Lehrkraft, vielmehr muss umgekehrt die Schule auf die Medien eingestellt werden. Allein das heute im Internet präsente lexikalische Wissen (mit der Encyclopedia Britannica als der letzten gefallenen Bastion des alten Systems) erlaubt nicht nur die eine beliebige Erhöhung des Schulwissens, sondern zugleich seine Kontrolle, unabhängig davon, wie ein Lehrerkonsens beschaffen ist. Ich könnte auch sagen, das Wissen demokratisiert sich, mindestens wird es vielfältig, umstritten und unabhängig von dem Format Buch. Kontrolle und Wissenserwerb fallen auseinander, Unterricht verliert seinen linearen Charakter, Lehren und Lernen übernehmen die Möglichkeiten des globalen Austausches, mit allen Konsequenzen für die Institution Schule, die sich nicht mehr auf den einen Lernort verlassen kann, an dem sie gegründet wurde und bislang gut überlebt hat. Die Lernorte selbst verändern sich, sie verlieren die Bindung an traditional definierte soziale Räume, weil sie auf Schnelligkeit reagieren müssen. Die stetige, sozusagen langsam fliessende Zeit der Schule, die sich genau erwarten liess, wird mit dem Lernmedium aufgesprengt. Forschendes Lernen im Internet lässt sich nicht im 45-Minuten-Takt bewerkstelligen, während in Zukunft Qualitätssteigerungen im Fachunterricht nur mit einem solchen Zugang möglich sein werden. Schulbücher sind viel zu langsam, um das Lerntempo halten zu können, an das sich die Schüler gewöhnen werden, auch weil ihnen keine andere Wahl bleibt.

Schnelligkeit ist die eine Seite, nicht zu bewältigende Vielfalt die andere. Computer und besonders die Internetzugänge bieten Speicherungen und Kommunikationen, die eine radikal neue Qualität definieren. Eine zentrale Frage wird sein, wie man diese Fülle angesichts der Erreichbarkeit innerhalb nützlicher Zeit bewältigt, wenn immer die meisten Anfragen nicht geschehen können. Es ist unmöglich, alle Suchergebnisse zum Thema Shakespeare auch nur anzuschauen, geschweige denn zu verarbeiten, während die Unsicherheit wächst, auf welche Quelle Verlass ist, was als Standardinterpretation gelten kann und wo das akut nützlichste Wissen zu finden ist. Die autoritäre Lehrerinterpretation hatte den unschätzbaren Vorteil, dass alle anderen Möglichkeiten entweder unerkannt blieben oder aber ausgeschlossen wurden. Im Internet gibt es $\mathrm{zu}$ jedem Thema des Schulwissens immer weit mehr Angebote, als eingelöst werden können, ohne dass Seriosität für Unterscheidung sorgte. Das Internet ist ein Transportmedium, das vollkommen liberal gehalten ist. Selbstzensur ist möglich, aber alle Schulbuchverlage, die Netzversionen ihrer Produkte anbieten, müssen nicht nur in Kauf nehmen, dass sich die Produkte völlig verändern, sondern sind zugleich einer Konkurrenz ausgesetzt, die nur über die Abnehmer kontrolliert wird. Die Anbieter werden nicht für unliebsame Produktkontrollen sorgen, daher werden Schulen Berater anstellen, die sich einzig damit beschäftigen, das mediale Angebot zu sortieren. Hinzukommt, dass die Abnehmer kontrollfähig werden. Was sollte Eltern daran hindern, die internationalen Lehrmittelangebote $\mathrm{zu}$ studieren und mit dem $\mathrm{zu}$ vergleichen, was die Schule ihres Kindes anzubieten hat?

Die Globalisierung der Bildung ist nicht vorhanden, sie entsteht. Und sie zwingt zu verschiedenen Konsequenzen, von denen nicht alle schön sind. Ich nenne nur:

1. Standardisierung des Angebots in den drei grossen Wirtschaftszonen Amerika, Japan und Europa.

2. Wettbewerb der Systeme und Evaluation der Resultate.

3. Kostendruck und Outsourcing.

4. Seriosität als knappes Gut.

5. Ungleichverteilung attraktiver Angebote.

«Globalisierung» der Bildung heisst nicht Individualisierung im Sinne der Freizeitindustrie, dafür sind schon die staatlichen Mittel viel zu hoch und sind die Investitionen viel zu riskant. Daher sind mehr oder weniger rigide Standardisierungen erwartbar, die der Bildung den Reiz des Individuellen nehmen sollen. Bildungsstandardisierungen sind für die drei zentralen Wirtschaftszonen des Globus erwartbar, also nicht oder nur nachgeordnet zu diesen in Bereichen der zweiten und dritten Welt. Der Wettbewerb der Systeme wird in den drei Zonen der ökonomischen Globalisierung aufgetragen und entschieden, mit kreativen, kleinen Aussenseitern, die gerade im Bereich der Bildungstechnologie ihre Chancen wahren werden. Die grossen Systeme, die flächendeckend versorgen und auf Legitimationsprobleme achten müssen, haben dann leicht einen Wettbewerbsnachteil, wie an der Rekrutierung von Computer-Fachleuten aus Indien abzulesen 
ist. Die grossen Systeme produzieren nicht spezifisch genug, weil sie mehr Aufgaben zu erfüllen haben als lediglich die Ausbildung für einen segmentierten und aber hochgradig lohnenden Arbeitsmarkt. Mit dem Druck, im weltweiten Wettbewerb das Richtige produzieren zu müssen, wächst die Unsicherheit, was wiederum eine Unmenge an Anbietern wachruft, die Marktchancen für Bildung sehen können. In dieser Situation wird Seriosität, seit dem 19. Jahrhundert staatlich garantiert, zum knappen Gut, und zwar weil Marktkräfte freigesetzt werden. Nunmehr wird es schwierig, Schulen auf annähernd gleichem Niveau attraktiv zu halten. Mit vermuteter Ungleichheit aber wird es einen Run geben auf knappe Angebote, die für besonders attraktiv gehalten werden, eine Entwicklung, die zum Beispiel durch das Maturiätsprivileg der Gymnasien oder den Tauschwert des Volksschulabschlusses lange gegengesteuert werden konnte.

Mein letzter Punkt geht auf die bildungspolitischen Folgen dieser Analyse ein. Wie sollen ortsgebundene Schulen auf globale Tendenzen reagieren, wenn diese Tendenzen wohl in ihren Auswirkungen gespürt werden, jedoch nicht Objekt sind, und zwar weder im Sinne einer erreichbaren Grösse noch im Sinne eines wirklichen Gegenstandes. Das Internet ist ein Transportmittel, kein Schulfach.

\section{Legitimation öffentlicher Bildung}

«Globalisierung» ist eine Raumvorstellung, aber wesentlich ein Zeitphänomen. Kennzeichnend ist wesentlich Gleichzeitigkeit, synchron, allenfalls knapp zeitverzögert, geschieht dasselbe, und zwar unabhängig von Lebensaltern, sozialen Rollen oder kultureller Zugehörigkeit. Neue Games von Nintendo werden zur gleichen Zeit eingeführt, allenfalls unterschieden nach den drei globalen Wirtschaftszonen, also etwas früher in den Vereinigten Staaten und Kanada als in Europa. Der national release von Hollywood-Produktionen bezieht sich inzwischen auf den angelsächsischen Weltmarkt und ist nur dadurch verzögert, dass sich das europäische Filmpublikum an Sprachsynchronisation gewöhnt hat. Das mit jeder grossen Produktion eng verbundene Merchandising hat nur als gleichzeitiges Angebot eine Verkaufschance, die sich erhöht, wenn möglichst alle angesprochen werden können, also die Produktpalette vom Kinderspielzeug bis einem Soundtrack reicht, der auch für Senioren interessant ist. Der jährliche Start der Disney-Weihnachtsanimation wird so vorbereitet, dass trotz der Synchronisation ein gleichzeitiger Start weltweit möglich ist, weil anders das Weihnachsgeschäft nicht organisiert werden könnte, das im
Übrigen ein Beispiel dafür ist, wie stabil ein sozialer Zeitstandard sein kann, soweit er sich kommerziell herausragend nutzen lässt.

Entscheidend für das globale Geschäft mindestens in den genannten Branchen ist Gleichzeitigkeit an möglichst vielen Orten. «Gleichzeitig» ist mit gleich verbunden, die Produkte müssen überall identisch sein, anders gäbe es keine Möglichkeit, sie kommerziell anzureizen. Sie verlieren daher jegliche Ortsbindung, selbst oder gerade Hollywood-Produktionen werden weltweit zusammengefügt, ohne dass auf das Qualitätslabel «Hollywood» verzichtet werden konnte. Der Ort wird virtuell, das Produkt entsteht aus globalen Kooperationen, während die Stars angelsächsisch sind und sich auf dem amerikanischen Markt bewähren müssen. Ich erwähne das, weil derartige Produktionen den Grossteil unserer ästhetischen Erfahrung bestimmen, also Bildung auf sehr wirksame Weise definieren können. Sie ist in diesem Sinn global, letztlich sorgt eine weltweite Hitparade für den Musikgeschmack von Kindern und Jugendlichen, prägt eine akute Mode die Selbstinszenierung und entscheidet ein Trend über Visualisierung, also das, was wir an Bildern akzeptieren und was nicht. Es ist paradox, dass diese Globalisierung mit weitgehend identischen Grundeinheiten, die je nur neu gemixt werden, als hochgradige Individualisierung wahrgenommen und agiert werden kann.

Bildung unterscheidet sich im Anspruch. Kommerzialisierung von Bildung heisst im wesentlichen Senkung der Ansprüche, weil Kundenmacht sich nicht oder nur schwach und lediglich in bestimmten Kaufsegmenten durch herausgehobene Standards steuern lässt. Kunden entscheiden nach Bedürfnissen und Bedarf, beides ohne einen grossen Planungshorizont, sieht man von der Lebensvorsorge einmal ab. Bedürfnisse werden möglichst kurzfristig realisiert, je nachdem, was das persönliche Einkommen zulässt, wobei auch hier Angebote die Grenzen verschieben. Nicht zufällig ist in der Tourismusindustrie von Last-Minute-Angeboten die Rede, die die Kurzfristigkeit nochmals komprimieren. Demgegenüber ist Bildung, mindestens in ihrer schulischen Form, ein langfristiges Geschäft, das einen Generationenvertrag voraussetzt. Dieser Vertrag ist formell nie geschlossen worden und gleichwohl ein erstrangiger gesellschaftlicher Tatbestand. Seit Beginn des 19. Jahrhunderts ist die Bildungsversorgung zu einer zunehmenden Selbstverständlichkeit geworden, die jede Lebensplanung bestimmt. Dabei sind steigende Niveaus in der Breite die Grunderfahrung, wesentlich bewirkt durch die flächendeckende Versorgung mit Volksschulen, die intensiv ausgebaut wurden und tatsächlich, soweit wir 
das historisch sagen können, für Niveausteigerung gesorgt haben. Die Voraussetzung war:

- staatliches Engagement und öffentliche Alimentierung

- gemeinsame Standards und vergleichbare Entwicklung

- der Aufbau und die Stabilisierung einer Profession

- Akzeptanz in der Gesellschaft mit der staatlichen Niveausicherung

- relative Autonomie der einzelnen Einheit

- unbefristete Überlebensgarantie.

Eine öffentliche Versorgung mit Volkschulen ist unabhängig davon nicht zu haben. Das historische System kann also nicht völlig anders gebaut werden, wenn es vergleichbare Effekte erzielen soll. Historisch besteht der Grundeffekt darin, ein steigendes Bildungsminimum garantieren zu können. Genau das macht den faktischen Generationenvertrag aus, er sieht nicht vor, dass am Ende alle das Gleiche können, sondern dass ein bestimmter Grundbestand an Wissen und Können, der im Niveau ständig angestiegen ist, für alle garantiert scheint. Wenn ich sage «scheint», dann aus zwei Gründen, Bildung ist kein automatisch gefertigtes Produkt, sondern ein fragiles Zusammenspiel aus sehr heterogenen Faktoren, die leicht auseinanderfallen können, und die Überzeugung und so die angenommene Notwendigkeit «Bildung» ist in bestimmten Hinsichten wichtiger als das je akute Resultat. Ich könnte auch sagen, entscheidend ist die Erwartung und daran anschliessend die Praxis. Wenigstens bestimmen Bildungsstandards sehr nachhaltig öffentliche Erwartungen, ich nenne nur

- Perfektionsgrade der Alphabetisierung

- Symbolverstehen in öffentlichen Räumen

- Wissensstandards in politischer Kommunikation

- eine persönliche Bildungspräsenz

- selbstverständliche Nutzung von Lernressourcen

- fortlaufende Strukturierung der Bildung

- Aushalten von Testsituationen etc.

Wer nicht Lesen oder Schreiben kann, und auch, wer dies in mangelnder Perfektion kann, wird die Internetrevolution nicht nutzen können. Die persönlichen Schwächen werden unmittelbar mit der Nutzung sichtbar, was auch ein noch so perfektes Fehlerprogramm nicht ausgeglichen kann, es sei denn, der Autor ist mit dem Fehlerprogramm identisch. Was man unschön «funktionale Analphabeten» nennt, ist gleichbedeutend mit der Erfahrung täglicher Behinderung in öffentlichen Räumen, die zunehmend rasches Symbolverstehen abverlangen. Ohne ein vorauszusetzendes Minimum an gemeinsamer Bildung wäre politische Kommunikation und so eine argumentativ orientierte Öffentlichkeit nicht möglich. Was der Ausfall von Bildung bewirkt, lässt sich leicht an der Macht von Demagogen ablesen, die dort emotionalisieren können, wo die Gewöhnung an den anspruchsvollen Austausch von möglichst guten Argumenten nicht vorhanden ist, wo, anders gesagt, politische Bildung nicht stattgefunden hat.

Erwartet wird aber immer auch eine persönliche Bildungspräsenz, also die Umsetzung der Bildungserfahrungen in eigenen Stil oder Kommunikationsfähigkeit. Schulen bereiten auch in diesem Sinne auf Bildungskulturen vor, die Unwissen oder Ignoranz sanktionieren. Man blamiert sich, wenn man den Faust für ein Drama von Schiller hält, das mit einem «zerbrochenen Krug» endet. Die Toleranz endet dort, wo sichtbares Nichtwissen nicht ausgeglichen wird, was dann die ständige Nutzung von Lernressourcen zu einer selbstverständlichen Erwartung werden lässt. Wer etwas nicht kann, ist gehalten, es zu lernen, und zwar als Reaktion auf die Verletzung der Erwartungen. Fortlaufendes Lernen ist nur möglich durch die ständige Restrukturierung der eigenen Bildung, also des Wissenshorizontes, mit dem man sich zu präsentieren gedenkt. Die Präsentation wird fortlaufend getestet, es gibt keine Projektplanung, kein Geschäftsessen und keine Konsumsituation ohne mitlaufende Wissenskontrollen, die die Kommunikation anderer danach testen, ob sie allgemeine Standards erfüllen oder nicht. Falsche Zahlen, schlechte Formulierungen, fehlende Bezüge, schiefe Kontexte sind negative Indikatoren für Bildung, die im Alltag hohe Relevanz erhalten. Man kann sozusagen umgekehrt zeigen, welche hohe Relevanz Bildung zugesprochen wird, unabhängig davon, wie der Computer genutzt wird.

Das gilt lokal wie global, es ist in Hongkong nicht wesentlich anders als in Hamburg, Unterschiede in der Höflichkeit oder im Taktverhalten beiseite gelassen. Die Bildungsverwendung kann daher nicht auf Jobspezialisierung verengt, sondern muss auf den Generationenvertrag bezogen werden, den die Internetrevolution nicht aufhebt, sondern verstärkt, wie immer anders die Zeitverhältnisse, die Lernformen oder die Art und Weise der Kommunikation in künftigen Schulen beschaffen sein mögen. Das Problem Bildung ist nicht dadurch gelöst, dass schnelle Zugänge und riesige Speicherungen bestehen. Den Umgang mit Computern lernen Kinder spielend, aber allein damit sind sie weder imstande, ein historisches Problem zu verstehen noch 
auf gesteigerte Ansprüche in der Mathematik zu reagieren. Mit dem Zugang zu riesigen Geographienetzen ist nicht garantiert, dass Kinder geographische Vorstellungen entwickeln, die leichte Erreichbarkeit lexikalischer Informationen gewährleistet nicht eine vertiefte Problemlösung, der ständige Kontakt mit Oberflächen sorgt nicht für den Aufbau von lernfähigen Wissensstrukturen. Genau diese Fachlichkeit muss gesichert werden, ein steigendes Minimum an inhaltlicher Bildungsversorgung für alle, anders lässt sich nicht mehr von öffentlicher Bildung sprechen, ganz unabhängig davon, wie sie sich in künftigen Informationsnetzen zu präsentieren versteht.

Entgegen dem Zeitgeist ist Präsentation nicht schon Bildung. Die Pointe ist, dass jede Präsentation mit persönlichen Bildungsindikatoren gelesen werden kann, anders wäre es unmöglich, Stufen oder Zonen des Gehaltvollen zu unterscheiden, also eine wirklich argumentative Arena von eitlem Geschwätz, eine überzeugende von einer niveaulosen Filmgeschichte oder eine informative Dokumention von einem Aufguss aus zweiter Hand. Wir können das vielfach ohne grosse Anstrengung, vermutlich wesentlich als Folge unablässiger Bildungssprozesse, die zur individualisierten Standardanwendung führen, ohne darin einen Widerspruch sehen $\mathrm{zu}$ müssen. Bildung macht nicht «gleich», sondern verlangt die persönliche Präsentation allgemeiner Standards, ohne dass diese starre Vorschriften wären. Die Folge dieser Einsicht ist, mit Modellen der Outputsteuerung vorsichtig zu sein, immer den grossen Rest an Individualisierung vor Augen zu haben und vom Prozess der Bildung anzunehmen, dass wesentlich ist, ihn fortsetzen zu können.

Bleibt die Frage, für welchen Arbeitsmarkt produzieren wir Bildung? Die heute verfügbaren Bildungstheorien verweigern sich zumeist dieser Frage, weil sie Bildung zweckfrei verstehen und wenigstens nicht einer ökonomischen Nutzung das Wort reden wollen. Aber natürlich stellt sich die Frage und sollte auch beantwortet werden. Ich habe fünf Punkte:

1. Bildung definiert die Niveaus der Lernfähigkeit und ist eine unverzichtbare Ressource.

2. Die Verwendung von Bildung ist an die lernende Person gebunden.

3. Der Arbeitsmarkt der Zukunft reagiert auf individuelle Kompetenz.

4. Die Kompetenz muss je neu auf Aufträge und Erwartungen eingestellt werden.

5. Die Nachfrage ist global, aber nur dann, wenn die Qualität nachgewiesen ist.
In diesem Sinne ist «Bildung» fortlaufende Qualitätssicherung, die eine grundlegende Initiation voraussetzt. Dafür sorgen Volksschulen, die also nicht etwa in ihrer Bedeutung abnehmen, sondern mit den neuen Medien eine Aufwertung erfahren, vorausgesetzt, es gelingt ihnen, Kompetenzen in den Mittelpunkt zu stellen, also nicht einfach Noten zu verteilen, sondern fachliche Qualität zu entwickeln, die sich überprüfen lässt. Entscheidend für den Arbeitsmarkt der Zukunft ist nicht die Note, sondern das Können in Französisch, globale Firmen verlangen perfektes Englisch, nicht eine «5 _», die mathematischen Berufe setzen Höhere Mathematik voraus, das Alltagsleben erwartet Alphabetisierungsstandards - die Volksschule wird sich daran messen lassen müssen, ob und wie weit sie diesen Erwartungen entgegenkommt. Sie bezieht sich indirekt auf einen Weltmarkt, auch wenn und vermutlich auch weil Schulorte, wie immer sie in Zukunft beschaffen sein mögen, sich nicht globalisieren können.

Was damit auf dem Prüfstand steht, ist die Grundidee einer liberalen öffentlichen Bildung, die deswegen auf die Volksschule übertragen wurde, weil alle Kinder, unabhängig vom sozialen Stand, vom Geschlecht, der Religion oder der ethnischen Zugehörigkeit am Prozess der Bildung teilhaben sollten. Das geschah nie ohne Benachteiligung, so jedoch, dass tatsächlich die durchschnittlichen Chancen wuchsen, die Bildungsbereitschaft mit der Zugänglichkeit von Chancen zunahm und Bildungsverweigerung zu einem Lebensrisiko wurde. Gemessen an diesen Kriterien kann Bildung nicht total individualisiert werden, ist das Marktprinzip nur begrenzt nützlich und bleibt die grundlegende Bildungsversorgung eine Aufgabe des Staates. Dies nicht, weil ein allgemein nützliches Produkt entsteht, sondern weil die Versorgung im Sockel der Volksschule garantiert sein muss. Im Gegenzug muss die Volksschule zeigen, dass und wie sie modernisierungsfähig ist, nur das verhindert eine feindliche Übernahme.

\section{Literatur}

Beisheim, M./Dreher, S./Walter, G./Zürn, M.: Globalisierung: Rhetorik oder Realität? Zum Stand der Denationalisierung in der G-7 und der Bundesrepublik. In: W. FRICKE (Hrsg.): Jahrbuch Arbeit und Technik. Bonn 1997, S. 96-180.

Bemmerlein, A. (Hrsg.): Das Sprachlabor in der Unterrichtspraxis. Ein Erfahrungsbericht des Schul- und Kulturreferats der Stadt Nürnberg. Freiburg i. Br. 1967. 
MedienPädagogik

Cuban, L.: Teachers and Machines. The Classroom Use of Technology Since 1920. New York/London: Teachers College Press 1986.

Elkins, D.: Beyond Sovereignity. Territory and Political Economy in the Twenty-first Century. Toronto 1995. Focus Behaim Globus. Teil 1: Aufsätze. Teil 2: Katalog. Bearb. v. J.K.W.

Willers u. a., Nürnberg 1992 (= Ausstellungskataloge des Germanischen Nationalmuseums, hrsg. v. G. BOTT)

Frieden, J.: Banking on the World. The Politics of American International Finance. New York 1988.

Habermas, J.: Die neue Unübersichtlichkeit. Kleine Politische Schriften V. Frankfurt am Main 1985.

Hirst, P/Thompson, G.: Globalization in Question. The International Economy and the Possibilities of Governance. Cambridge: Cambridge University Press 1996.

Komensky, J.A.: Allgemeine Beratung über die Verbesserung der menschlichen Dinge. Hrsg. u. übers. v. F. HOFMANN. Berlin 1970. (= Pädagogische Bibliothek, hrsg.v. K.-H. GÜNTHER/H.KÖNIG)

Oelkers, J.: Der Verlust der Hohen Warte. Ms. Zürich 1999.

Othmae, K.: Triad Power. The Coming Shape of Global Competition. New York 1985.

Scholte, J.A.: Beyond the Buzzword. Toward a Critical Theory of Globalization. In: E. Kofman/G. Youngs (Eds.): Globalization. Theory and Practice. London 1996, S. 43-57.

Zymek, B.: «Leitbild ist nicht mehr der erwerbstätige, sondern der tätige Mensch.»Ein bildungshistorischer Kommentar zu den Forderungen der Kommissionen für Zukunftsfragen der Freistaaten Bayern und Sachsen. In: Zeitschrift für Pädagogik 44, 6 (1998), S. 789-803. 